\title{
Anti-periodic extremal problems for a class of nonlinear evolution inclusions in $R^{N}$
}

\author{
Jingrui Zhang ${ }^{1 *}, \mathrm{Yi}_{\mathrm{i}}$ Cheng ${ }^{2}$, Cuiying $\mathrm{Li}^{3}$ and Hongtu Hua ${ }^{4}$
}

${ }^{*}$ Correspondence:

jingruizhang123@126.com.cn

'School of Aerospace Engineering, Beijing Institute of Technology,

Beijing, 100081, P.R. China

Full list of author information is

available at the end of the article

\begin{abstract}
The aim of this paper is to establish the existence of anti-periodic solutions to the following nonlinear anti-periodic problem: $\dot{x}+A(t, x) \in \operatorname{Ext} F(t, x)$ a.e. $t \in l, x(T)=-x(0)$, in $R^{N}$ where $\operatorname{Ext} F(t, x)$ denotes the extremal point set of the multifunction $F(t, x)$, and $A(t, x)$ is a nonlinear map from $R^{N}$ to $R^{N}$. Sufficient conditions for the existence of extremal solutions are presented. Also, we prove that the extremal point set of this problem is compact in $C\left(I, R^{N}\right)$ and dense in the solution set of nonlinear evolution problems with a convex valued perturbation which is multivalued. We apply our results on the control system with a priori feedback.
\end{abstract}

Keywords: anti-periodic solution; evolution inclusion; the Schauder fixed point theorem; continuous selection

\section{Introduction}

In this paper, we consider the following anti-periodic problems:

$$
\left\{\begin{array}{l}
\dot{x}+A(t, x) \in \operatorname{Ext} F(t, x) \quad \text { a.e. } t \in I, \\
x(T)=-x(0)
\end{array}\right.
$$

in $R^{N}$ where $I=[0, T], F(t, x): I \times R^{N} \rightarrow 2^{R^{N}}$ satisfies some conditions mentioned later, $A(t, x)$ is a nonlinear map from $I \times R^{N}$ to $R^{N}$. Anti-periodic problems of evolution inclusions have important applications in many fields, such as auto-control, partial differential equations and engineering, etc. The study of anti-periodic solutions for nonlinear evolution equations was initiated by Okochi [1]. Since then, many authors devoted themselves to the investigation of the existence of anti-periodic solutions to nonlinear evolution equations in Hilbert spaces. For the details, see [2-7] and the references therein. In [5], Chen studied the anti-periodic solution for the following first order semilinear evolution equation:

$$
\left\{\begin{array}{l}
\dot{u}+A u(t) \in F(t, u), \quad t \in R \\
u(t+T)=-u(t)
\end{array}\right.
$$

where $A: R^{N} \rightarrow R^{N}$ is a matrix, $f: R \times R^{N} \rightarrow R^{N}$ is a continuous function satisfying $f(t+T, u)=-f(t,-u)$ for all $(t, u) \in R \times R^{N}$. Chen et al. [8] also studied this problem in a real separable Hilbert space when $A$ is a dense self-adjoint operator which only has a point spectrum. Recently, Q Liu [9], ZH Liu [10] and Wang [11] considered anti-periodic problem of nonlinear evolution equation in a real reflexive Banach space and obtained some

\section{Springer}

○2014 Zhang et al.; licensee Springer. This is an Open Access article distributed under the terms of the Creative Commons Attribution License (http://creativecommons.org/licenses/by/2.0), which permits unrestricted use, distribution, and reproduction in any medium, provided the original work is properly cited. 
existence results by applying the theory of pseudo-monotone perturbations of maximal monotone mappings and the theory of evolution operators. However, there are few results about the extremal anti-periodic problem, which is connected with the 'Bang-Bang' control problem in the above works. For the case of periodic problems, we refer to the work of Li-Xue [12], Xue-Yu [13] in Hilbert and Xue-Cheng [14] in Banach space. Inspired by [15], which considers the problem in Banach space, we continue to consider the existence of solutions for a nonlinear evolution inclusion in $R^{N}$ by relaxing the constraint conditions. Our approach will be based on techniques and results of the theory of the extremal continuous selection theorem and the Schauder fixed point theorem.

The paper is divided into four parts. In Section 2, we introduce some notation, and definitions we need for the results. In Section 3, we present some basic assumptions and main results, the proofs of the main results are given based on the Schauder fixed point theorem and the extremal continuous selection theorem. Finally, an example is presented for our results in Section 4.

\section{Preliminaries}

For convenience, we introduce some notation as follows. In Euclidean space, $(\cdot, \cdot)$ expresses inner product, while $\|\cdot\|$ expresses the Euclidean norm. Let $L^{2}\left([0, T] ; R^{N}\right)$ denote the set of the map $x:[0, T] \rightarrow R^{N}$ which satisfies $\int_{0}^{T}|x|^{2} d t<\infty$, and the norm in $L^{2}\left([0, T] ; R^{N}\right)$ is denoted by $\|x\|_{2}=\left(\int_{0}^{T}|x|^{2} d t\right)^{\frac{1}{2}}$. If $F=[0, b]$, the $\operatorname{Ext} F=\{0, b\}$. We recall some basic definitions and facts from multivalued analysis which we shall need in the sequel. For details we refer to the book of Zeidler [16]. Let $I=[0, T],(I, \Sigma)$ be the Lebesgue measurable space and $X$ be a separable Banach space. Denote

$$
\begin{aligned}
& P_{f}(X)=\{A \subset X: \text { nonempty and closed }\} \\
& P_{k}(X)=\{A \subset X: \text { nonempty and compact }\} \\
& P_{w k c}(X)=\{A \subset X: \text { nonempty, weakly compact and convex }\} .
\end{aligned}
$$

Let $A \subset P_{f}(X), x \in X$, then the distance from $x$ to $A$ is given by $d(x, A)=\inf \{|x-a|: a \in A\}$. A multifunction $F: I \rightarrow P_{f}(X)$ is said to be measurable if and only if, for every $z \in X$, the function $t \rightarrow d(z, F(t))=\inf \{\|z-x\|: x \in F(t)\}$ is measurable. A multifunction $G: I \rightarrow$ $2^{X} \backslash\{\emptyset\}$ is said to be graph measurable, if $\operatorname{Gr} G=\{(t, x): x \in G(t)\} \in \Sigma \times \mathscr{B}(X)$ with $\mathscr{B}(X)$ being the Borel $\sigma$-field of $X$. On $P_{f}(X)$ we can define a generalized metric, known in the literature as the 'Hausdorff metric', by setting

$$
h(A, B)=\max \left\{\sup _{a \in A} d(a, B), \sup _{b \in B} d(b, A)\right\}
$$

for all $A, B \in P_{f}(X)$. It is well known that $\left(P_{f}(X), h\right)$ is a complete metric space and $P_{f c}(X)$ is a closed subset of it. When $Z$ is a Hausdorff topological space, a multifunction $G: Z \rightarrow$ $P_{f}(X)$ is said to be $h$-continuous if it is continuous as a function from $Z$ into $\left(P_{f}(X), h\right)$.

Let $Y, Z$ be Hausdorff topological spaces and $G: Y \rightarrow 2^{Z} \backslash\{\phi\}$. We say that $G(\cdot)$ is 'upper semicontinuous (USC)' (resp., 'lower semicontinuous (LSC)'), if for all $C \subseteq Z$ nonempty closed, $G^{-}(C)=\{y \in Y: G(y) \cap C \neq \phi\}$ (resp., $G^{+}(C)=\{y \in Y: G(y) \subseteq C\}$ ) is closed in $Y$. An USC multifunction has a closed graph in $Y \times Z$, while the converse is true if $G$ is locally compact (i.e. for every $y \in Y$ there exists a neighborhood $U$ of $y$ such that $\overline{F(U)}$ is compact 
in Z). A multifunction which is both USC and LSC is said to be 'continuous'. If $Y, Z$ are both metric spaces, then the above definition of LSC is equivalent to saying that for all $z \in Z, y \rightarrow d_{Z}(z, G(y))=\inf \left\{d_{Z}(z, v): v \in G(y)\right\}$ has upper semicontinuity as an $R_{+}$-valued function. Also, lower semicontinuity is equivalent to saying that if $y_{n} \rightarrow y$ in $Y$ as $n \rightarrow \infty$, then

$$
\begin{aligned}
G(y) & \subseteq \underline{\lim } G\left(y_{n}\right)=\left\{z \in Z: \lim d_{Z}\left(z, G\left(y_{n}\right)\right)=0\right\} \\
& =\left\{z \in Z: z=\lim z_{n}, z_{n} \in G\left(y_{n}\right), n \geq 1\right\} .
\end{aligned}
$$

A set $D \subseteq L^{2}(I, X)$ is said to be 'decomposable', if for every $g_{1}, g_{2} \in D$ and for every $J \subseteq I$ measurable we have $\chi_{J} g_{1}+\chi_{J^{c}} g_{2} \in D$. Let $X$ be a Banach space and let $L^{2}(I, X)$ be the Banach space of all functions $u: I \rightarrow X$, which are Bochner integrable. $D\left(L^{2}(I, X)\right)$ denotes the collection of nonempty decomposable subsets of $L^{2}(I, X)$. The following lemmas are still needed in the proof of our main theorems.

Lemma 2.1 (see [17]) Let $X$ be a separable metric space and let $F: X \rightarrow D\left(L^{2}(I, X)\right)$ be a lower semicontinuous multifunction with closed decomposable values. Then $F$ has a continuous selection.

Let $X$ be a separable Banach Space and $C(I, X)$ be the Banach space of all continuous functions. A multifunction $F: I \times X \rightarrow P_{w k c}(X)$ is said to be Carathéodory type, if for every $x \in X, F(\cdot, x)$ is measurable, and for almost all $t \in I, F(t, \cdot)$ is $h$-continuous. (i.e. it is continuous form $X$ to the metric space $\left(P_{f}(X), h\right)$ where $h$ is Hausdorff metric). Let $M \subset$ $C(I, X)$. A multifunction $F: I \times X \rightarrow P_{w k c}(X)$ is called integrably bounded on $M$ if there exists a function $\lambda: I \rightarrow R_{+}$such that for almost all $t \in I$, $\sup \{\|y\|: y \in F(t, x(t)), x(\cdot) \in M\} \leq$ $\lambda(t)$. A nonempty subset $M_{0} \subset C(I, X)$ is called $\sigma$-compact if there is a sequence $\left\{M_{k}\right\}_{k \geq 1}$ of compact subsets $M_{k}$ such that $M_{0}=\bigcup_{k \geq 1} M_{k}$. Let $M_{0} \subset M$, such that $M_{0}$ is dense in $M$ and $\sigma$-compact. The following continuous selection theorem in the extreme point case is due to Tolstonogov [18].

Lemma 2.2 (see [18]) Let the multifunction $F: I \times X \rightarrow P_{w k c}(X)$ be of Carathéodory type and integrably bounded. Then there exists a continuous function $g: M \rightarrow L_{p}(I, X)$ such that for almost all $t \in I$, if $x(\cdot) \in M_{0}$, then $g(x)(t) \in \operatorname{Ext} F(t, x(t))$, and if $x(\cdot) \in M \backslash M_{0}$, then $g(x)(t) \in \overline{\operatorname{Ext}} F(t, x(t))$.

\section{Main results}

Let $I=[0, T]$ and $C\left(I ; R^{N}\right)$ be all the continuous functions from $I$ to $R^{N}$ with the max norm. We let $C_{\beta}=\left\{v(\cdot) \in C\left(I ; R^{N}\right): v(0)=-v(T)\right\}$, and $W^{1,2}\left(I ; R^{N}\right)=\left\{u(\cdot) \in C_{\beta}: \dot{u}(\cdot) \in L^{2}\left(I ; R^{N}\right)\right\}$. $W^{1,2}\left(I ; R^{N}\right)$ is a separable Banach space under the norm $\|\cdot\|_{1,2}$.

Consider the following anti-periodic problem:

$$
\left\{\begin{array}{l}
\dot{x}(t)+A(t, x(t)) \in \operatorname{Ext} F(t, x(t)) \quad \text { a.e. } t \in I \\
x(0)=-x(T)
\end{array}\right.
$$

where $A: I \times R^{N} \rightarrow R^{N}$ is a hemicontinuous function, $F: I \times R^{N} \rightarrow 2^{R^{N}}$ is a multifunction. By a solution $x$ of problem (1), we mean a function $x \in W^{1,2}\left(I, R^{N}\right)$ and there exists a 
function $f(t) \in \operatorname{Ext} F(t, x(t))$ such that

$$
\langle\dot{x}(t), v\rangle+\langle A(t, x(t)), v\rangle=\langle f(t), v\rangle
$$

for all $v \in R^{N}$ and almost all $t \in I$. The precise hypotheses on the data of problem (1) are the following:

$H(A): A: I \times R^{N} \rightarrow R^{N}$ is a nonlinear function such that

(i) $t \rightarrow A(t, x)$ is measurable;

(ii) for each $t \in I$, the operator $A(t, \cdot): R^{N} \rightarrow R^{N}$ is uniformly monotone and hemicontinuous, that is, there exists a constant $p>0$ such that $\left(A\left(t, x_{1}\right)-A\left(t, x_{2}\right), x_{1}-x_{2}\right) \geq p\left\|x_{1}-x_{2}\right\|^{2}$ for all $x_{1}, x_{2} \in R^{N}$.

$H(F): F: R \times R^{N} \rightarrow P_{k c}\left(R^{N}\right)$ is a multifunction such that

(i) $(t, x) \rightarrow F(t, x)$ is graph measurable;

(ii) for almost all $t \in I, x \rightarrow F(t, x)$ is $h$-continuous;

(iii) there exist a nonnegative function $b(\cdot) \in L_{+}^{2}(I)$ and a constant $c_{1}>0$ such that

$$
|F(t, x)|=\sup \{\|f\|: f \in F(t, x)\} \leq b(t)+c\|x\|^{\alpha}
$$

for all $x \in R^{N}, t \in T$, where $\alpha<1$.

We still need the following lemma.

Lemma 3.1 (see [19]) If the hypothesis $H(A)$ holds, consider the equation

$$
\dot{x}+A(t, x)=f(t) \quad \text { a.e. } t \in I,
$$

where $f \in L^{2}\left([0, T] ; R^{N}\right)$. Then problem (2) has a unique T-anti-periodic solution.

Theorem 3.1 If hypotheses $H(A)$ and $H(F)$ hold, then problem (1) has at least one solution.

Proof We define $L: W^{1,2}\left(I ; R^{N}\right) \rightarrow L^{2}\left([0, T] ; R^{N}\right)$ as $L x=\dot{x}+A(t, x)$ and $x(0)=-x(T)$. By Lemma 3.1, we have $L: W^{1,2}\left(I ; R^{N}\right) \rightarrow L^{2}\left([0, T] ; R^{N}\right)$ is one to one and surjective, and so $L^{-1}: L^{2}\left([0, T] ; R^{N}\right) \rightarrow W^{1,2}\left(I ; R^{N}\right)$ is well defined. As in the proof of Theorem 3.1, we obtain a priori bound for $S_{e}$ which denotes the solution set of problem (1). We know that there exist $M_{i}>0, i=1,2$, such that $\|x\|_{1,2}<M_{1}$ and $\|x\|_{C(I, H)}<M_{2}$ for all $x \in S_{e}$. Let $\psi(t)=$ $b(t)+C M_{2}, \psi(t) \in L_{2}^{+}(I)$. We may assume that $|F(t, x)| \leq \psi(t)$, a.e. on $I$ for all $x \in R^{N}$. So let

$$
W=\left\{v \in L_{q}(I, H):\|v(t)\|_{H} \leq \psi(t) \text { a.e. on } I\right\},
$$

then $\hat{K}=L^{-1}(W) \subseteq W^{1,2}\left(I ; R^{N}\right)$ is compact convex subset in $C(I, H)$. Obviously $\hat{K}$ is convex. We only need to show the compactness. Let $\left\{x_{n}\right\}_{n \geq 1} \subset \hat{K}$, then there exists $h_{n} \in W$ such that $L\left(x_{n}\right)=h_{n}$, i.e. $\dot{x}_{n}=h_{n}-A\left(t, x_{n}\right)$. By the definition of $W$, so $W$ is uniformly bounded in $L^{2}\left([0, T] ; R^{N}\right)$. By the Dunford-Pettis theorem, passing to a subsequence if necessary, we may assume that $h_{n} \rightarrow h$ in $L^{2}\left([0, T] ; R^{N}\right)$ for some $h \in W$. From the definition of $W$, we have

$$
\left\|x_{n}\right\|_{W_{1,2}}=\left\|L^{-1}\left(L x_{n}\right)\right\|_{W_{1,2}}=\left\|L^{-1} h_{n}\right\|_{W_{1,2}} \leq M_{1} .
$$


Therefore, the sequence $\left\{x_{n}\right\}_{n \geq 1} \subset W^{1,2}\left(I ; R^{N}\right)$ is bounded. Because of the compactness of the embedding $W^{1,2}\left(I ; R^{N}\right) \subset L^{2}\left([0, T] ; R^{N}\right)$, we find that the sequence $\left\{x_{n}\right\}_{n \geq 1} \subset$ $L^{2}\left([0, T] ; R^{N}\right)$ is relatively compact. So by passing to a subsequence if necessary, we may assume that $x_{n} \rightarrow x$ in $L^{2}\left([0, T] ; R^{N}\right)$. Moreover, by the boundedness of the sequence $\left\{x_{n}\right\}_{n \geq 1} \subset W^{1,2}\left(I ; R^{N}\right)$, it follows that the sequence $\left\{\dot{x}_{n}\right\}_{n \geq 1} \subset L^{2}\left([0, T] ; R^{N}\right)$ is uniformly bounded and passing to a subsequence if necessary, we may assume that $\dot{x}_{n} \rightarrow \dot{x}$ in $L^{2}\left([0, T] ; R^{N}\right)$. Since the embedding $W^{1,2}\left(I ; R^{N}\right) \subset C\left(I ; R^{N}\right)$ is continuous and $W^{1,2}\left(I ; R^{N}\right) \subset L^{2}\left([0, T] ; R^{N}\right)$ is compact, it follows that $x_{n} \rightarrow x$ in $C\left(I ; R^{N}\right)$ and $x_{n} \rightarrow x$ in $L^{2}\left([0, T] ; R^{N}\right)$. Hence, $x_{n} \rightarrow x$ in $R^{N}$ for all $t \in I \backslash \Lambda, m(\Lambda)=0$ ( $m$ being the Lebesgue measure on $R$ ). Since $A$ is hemicontinuous and monotone. Thus, $A\left(t, x_{n}\right) \rightarrow A(t, x)$ in $L^{2}\left([0, T] ; R^{N}\right)$ and as $n \rightarrow \infty$, we obtain $\dot{x}+A(t, x)=h$ a.e. on $I$ and $x(0)=-x(T)$. Note that

$$
\dot{x}_{n}-\dot{x}+\left(A\left(t, x_{n}\right)-A(t, x)\right)=h_{n}-h .
$$

Taking the inner product above with $x_{n}-x$ and integrating from 0 to $T$, one can see that

$$
\begin{aligned}
& \int_{0}^{T}\left(A\left(t, x_{n}\right)-A(t, x), x_{n}-x\right) d t \\
& \quad=\int_{0}^{T}\left(h_{n}-h, x_{n}-x\right) d t-\int_{0}^{T}\left(\dot{x}_{n}-\dot{x}, x_{n}-x\right) d t \\
& \quad \leq 2 \int_{0}^{T}\|\psi\|\left\|x_{n}-x\right\| d t \\
& \quad \leq 2\|\psi\|_{L^{2}}\left\|x_{n}-x\right\|_{L^{2}} \rightarrow 0 \quad \text { as } n \rightarrow \infty .
\end{aligned}
$$

By hypothesis $H(A)$, it follows that

$$
\begin{aligned}
\int_{0}^{T}\left(A\left(t, x_{n}\right)-A(t, x), x_{n}-x\right) d t & \geq p \int_{0}^{T}\left\|x_{n}-x\right\|^{2} d t \\
& \rightarrow 0 \quad \text { as } n \rightarrow \infty .
\end{aligned}
$$

So, we can find $\tau \in I \backslash \Lambda$ such that

$$
\left\|x_{n}(\tau)-x(\tau)\right\| \rightarrow 0 \quad \text { as } n \rightarrow \infty
$$

Using the integration by parts formula for functions in $W^{1,2}\left(I ; R^{N}\right)$, for any $t \in I$ we have

$$
\begin{aligned}
\left\|x_{n}(t)-x(t)\right\|^{2} & =\left\|x_{n}(\tau)-x(\tau)\right\|^{2}+2 \int_{\tau}^{t}\left(\dot{x}_{n}(s)-\dot{x}(s), x_{n}(s)-x(s)\right) d s \\
& \leq\left\|x_{n}(\tau)-x(\tau)\right\|^{2}+4\|\varphi(t)\|_{L^{2}}\left\|x_{n}(t)-x(t)\right\|_{L^{2}} .
\end{aligned}
$$

By (5), we see that

$$
\max _{t \in I}\left\|x_{n}(t)-x(t)\right\| \rightarrow 0 \quad \text { as } n \rightarrow \infty .
$$


So, $x_{n}(t) \rightarrow x(t)$ in $C\left(I ; R^{N}\right)$. Since $x=L^{-1}(h)$ with $h \in W$, we conclude that $L^{-1}(W) \subseteq$ $C\left(I ; R^{N}\right)$ is compact. From Lemma 2.2, we can find a continuous map $f: \hat{K} \rightarrow L^{2}\left(I ; R^{N}\right)$ such that $f(x)(t) \in \operatorname{Ext} F(t, x(t))$ a.e. on $I$ for all $x \in \hat{K}$. Then $L^{-1} \circ f$ is a compact operator. On applying the Schauder fixed point theorem, there exists a $x \in \hat{K}$ such that $x=L^{-1} \circ f(x)$. This is a solution of $(1)$, and so $S_{e} \neq \emptyset$ in $W^{1,2}\left(I ; R^{N}\right)$.

For the relation theorem of problem (1), we need the following definition and hypotheses.

Definition 3.1 (see [20]) The multifunction mapping $F: I \times R^{N} \rightarrow P_{k}\left(R^{N}\right)$ is called 'onesided Lipschitz (OSL)' continuous if there is an integrable function $\omega: I \rightarrow R_{+}$such that for every $x, y \in R^{N}, t \in I$, and $v \in F(t, x)$ there exists $u \in F(t, y)$ such that

$$
(v-u, x-y) \leq \omega(t)\|x-y\|^{2} .
$$

Under the above hypotheses, let $S$ denote the solution set of the equation $\dot{x}(t)+$ $A(t, x(t)) \in F(t, x), x(0)=-x(T)$.

Theorem 3.2 If hypotheses $H(A)$ and $H(F)$ hold and, moreover, $F(t, x)$ satisfies the OSL condition, we have $\overline{S_{e}}=S$, where the closure is taken in $C\left(I ; R^{N}\right)$.

Proof Let $x \in S$, then there exist $f \in L^{2}\left(I ; R^{N}\right)$ and $f(x)(t) \in F(t, x(t))$ a.e. on $I$, such that

$$
\begin{aligned}
& \dot{x}(t)+A(t, x(t))=f(t, x), \\
& x(0)=-x(T) .
\end{aligned}
$$

As before let $W=\left\{v \in L^{2}\left(I ; R^{N}\right):\|v\| \leq \psi(t)\right.$ a.e. on $\left.I\right\}$, then $\hat{K}=L^{-1}(W) \subseteq W^{1,2}\left(I ; R^{N}\right)$ is compact convex subset in $C\left(I ; R^{N}\right)$. For every $y \in \hat{K}$, we define the multifunction

$$
Q_{\epsilon}(t)=\left\{v \in F(t, y):(f-v, x-y) \leq \omega(t)\|x-y\|^{2}+\epsilon\right\} .
$$

Clearly, for every $t \in I, Q_{\epsilon}(t) \neq \emptyset$, and it is graph measurable. On applying the Aumann selection theorem, we get a measurable function $v: I \rightarrow R^{N}$ such that $v(t) \in Q_{\epsilon}(t)$ almost everywhere on $I$. So we define the multifunction

$$
R_{\epsilon}(y)=\left\{v \in S_{F(\cdot, y)}:(f-v, x-y) \leq \omega(t)\|x-y\|^{2}+\epsilon\right\} .
$$

We see that $R_{\epsilon}: \hat{K} \rightarrow 2^{L^{2}\left(I ; R^{N}\right)}$ has nonempty and decomposable values. It follows from Theorem 3 of [21] that $\overline{R_{\epsilon}(\cdot)}$ is LSC. Therefore $y \rightarrow \overline{R_{\epsilon}(y)}$ is LSC and has closed and decomposable values. So we apply Lemma 2.1 to get a continuous map $f_{\epsilon}: \hat{K} \rightarrow L^{2}\left(I ; R^{N}\right)$ such that $f_{\epsilon}(y) \in \overline{R_{\epsilon}(y)}$ for all $y \in \hat{K}$. Invoking II-Theorem 8.31 of [22] (in [22, p.260]), we can find a continuous map $g_{\epsilon}: \hat{K} \rightarrow L^{2}\left(I ; R^{N}\right)$ such that $g_{\epsilon}(y)(t) \in \operatorname{Ext} F(t, y)$ almost everywhere on $I$, and $\left\|f_{\epsilon}(y)-g_{\epsilon}(y)\right\| \leq \epsilon$ for all $y \in \hat{K}$. Now let $\epsilon \rightarrow 0$ and set $f_{\epsilon_{n}}=f_{\epsilon}, g_{\epsilon_{n}}=g_{\epsilon}$. Note that $\left\|g_{\epsilon_{n}}(y)\right\| \leq \psi(t)$ a.e. on $I$ with $\psi \in L^{2}\left(I ; R^{N}\right)$, so we have $g_{\epsilon_{n}} \rightarrow f_{\epsilon_{n}}$ in $L^{2}\left(I ; R^{N}\right)$. We consider the following problem:

$$
\begin{aligned}
& \dot{x}(t)+A(x)(t)=g_{\epsilon_{n}}(x)(t), \\
& x(0)=-x(T),
\end{aligned}
$$


where $g_{\epsilon_{n}}(x) \in \operatorname{Ext} R_{\epsilon}(x)$. We see that $L^{-1} g_{\epsilon_{n}}: \hat{K} \rightarrow \hat{K}$ is a compact operator and by the Schauder fixed point theorem, we obtain a solution $x_{\epsilon_{n}} \in S_{e} \subset W^{1,2}\left(I ; R^{N}\right)$ of (7). We see that the sequence $\left\{x_{\epsilon_{n}}\right\}_{n \geq 1} \subset \hat{K}$ is uniformly bounded. So by passing to a subsequence if necessary, we may assume that $x_{\epsilon_{n}} \rightarrow \hat{x}$ in $W^{1,2}\left(I ; R^{N}\right)$. From the proof of Theorem 3.1, we know that $x_{\epsilon_{n}} \rightarrow \hat{x}$ in $C(I, H)$ and $\hat{x}(0)=-\hat{x}(T)$. Note that $L x_{\epsilon_{n}}-L x=g_{\epsilon_{n}}\left(x_{\epsilon_{n}}\right)-f(x)$. So, we have

$$
\begin{aligned}
& \left(\dot{x}_{\epsilon_{n}}(t)-\dot{x}(t), x_{\epsilon_{n}}(t)-x(t)\right)+\left(A\left(x_{\epsilon_{n}}\right)(t)-A(x)(t), x_{\epsilon_{n}}(t)-x(t)\right) \\
& \quad=\left(g_{\epsilon_{n}}\left(x_{\epsilon_{n}}\right)(t)-f(x)(t), x_{\epsilon_{n}}(t)-x(t)\right) .
\end{aligned}
$$

However,

$$
\left(A\left(x_{\epsilon_{n}}\right)(t)-A(x)(t), x_{\epsilon_{n}}(t)-x(t)\right) \geq 0 \quad \text { a.e. } I .
$$

Then,

$$
\begin{aligned}
& \left(\dot{x}_{\epsilon_{n}}(t)-\dot{x}(t), x_{\epsilon_{n}}(t)-x(t)\right) \\
& \quad \leq\left(g_{\epsilon_{n}}\left(x_{\epsilon_{n}}\right)(t)-f(x)(t), x_{\epsilon_{n}}(t)-x(t)\right) \\
& \quad=\left(g_{\epsilon_{n}}\left(x_{\epsilon_{n}}\right)(t)-f_{\epsilon_{n}}\left(x_{\epsilon_{n}}\right)(t), x_{\epsilon_{n}}(t)-x(t)\right)+\left(f_{\epsilon_{n}}\left(x_{\epsilon_{n}}\right)(t)-f(x)(t), x_{\epsilon_{n}}(t)-x(t)\right) .
\end{aligned}
$$

By $g_{\epsilon_{n}} \rightarrow f_{\epsilon_{n}}$ in $L^{2}\left(I ; R^{N}\right)$ and $x_{\epsilon_{n}} \rightarrow \hat{x}$ in $L^{2}\left(I ; R^{N}\right)$, we have

$$
\begin{aligned}
& \left(g_{\epsilon_{n}}\left(x_{\epsilon_{n}}\right)(t)-f_{\epsilon_{n}}\left(x_{\epsilon_{n}}\right)(t), x_{\epsilon_{n}}(t)-x(t)\right) \\
& =\left(g_{\epsilon_{n}}\left(x_{\epsilon_{n}}\right)(t)-f_{\epsilon_{n}}\left(x_{\epsilon_{n}}\right)(t), x_{\epsilon_{n}}(t)-\hat{x}(t)\right) \\
& \quad+\left(g_{\epsilon_{n}}\left(x_{\epsilon_{n}}\right)(t)-f_{\epsilon_{n}}\left(x_{\epsilon_{n}}\right)(t), \hat{x}(t)-x(t)\right) \rightarrow 0 \quad \text { a.e. } I .
\end{aligned}
$$

Hence, there exists a constant $N_{0}>0$, and one has

$$
\left|\left(g_{\epsilon_{n}}\left(x_{\epsilon_{n}}\right)(t)-f_{\epsilon_{n}}\left(x_{\epsilon_{n}}\right)(t), x_{\epsilon_{n}}(t)-x(t)\right)\right|<\epsilon
$$

as $n>N_{0}$. It follows that

$$
\begin{aligned}
\frac{1}{2} \frac{d}{d t}\left\|x_{\epsilon_{n}}-x\right\|^{2}= & \left(\dot{x}_{\epsilon_{n}}(t)-\dot{x}(t), x_{\epsilon_{n}}(t)-x(t)\right) \\
\leq & \left(g_{\epsilon_{n}}\left(x_{\epsilon_{n}}\right)(t)-f_{\epsilon_{n}}\left(x_{\epsilon_{n}}\right)(t), x_{\epsilon_{n}}(t)-x(t)\right) \\
& +\left(f_{\epsilon_{n}}\left(x_{\epsilon_{n}}\right)(t)-f(x)(t), x_{\epsilon_{n}}(t)-x(t)\right) \\
\leq & \left(f_{\epsilon_{n}}\left(x_{\epsilon_{n}}\right)(t)-f(x)(t), x_{\epsilon_{n}}(t)-x(t)\right)+\epsilon \\
\leq & \omega(t)\left\|x_{\epsilon_{n}}-x\right\|^{2}+\epsilon .
\end{aligned}
$$

Let $S_{n}(t)=\left\|x_{\epsilon_{n}}-x\right\|^{2}$, then $\dot{S}_{n}(t) \leq 2 \omega(t) S_{n}(t)+2 \epsilon$. Integrating over (9) from 0 to $t$, one has

$$
S_{n}(t) \leq \int_{0}^{t} 2 \omega(s) S_{n}(s) d s+S(0)+2 \epsilon t .
$$


By using the Gronwall inequality, we have

$$
S_{n}(t) \leq \int_{0}^{t} 2 \omega(s)\left(S_{n}(0)+2 \epsilon s\right) \exp ^{2 \int_{0}^{t} \omega(\mu) d \mu} d s+S_{n}(0)+2 \epsilon t .
$$

From $g_{\epsilon_{n}}(x)(t) \in F(t, x)$, we know that $S_{n}(0)=0$. Let $\epsilon \rightarrow 0$, we have $S_{n}(t) \rightarrow 0$, i.e. $\| x_{\epsilon}(t)-$ $x(t) \| \rightarrow 0$ for any $t \in I$. Therefore, $x=\hat{x}$, i.e. $x_{\epsilon} \rightarrow x$ and $x_{\epsilon} \in S_{e}$, and so $S \subseteq \overline{S_{e}}$. Also $S$ is closed in $C(I, H)$ (see the proof of Theorem 3.1), thus $S=\overline{S_{e}}$.

\section{An application}

We present an example of a nonlinear anti-periodic distributed parameter control system, with a priori feedback (i.e. state dependent control constraint set). Let $T=[0, b]$, $\dot{x}=\left(\dot{x}_{1}, \dot{x}_{2}, \ldots, \dot{x}_{N}\right)$. Consider the following control system:

$$
\left\{\begin{array}{l}
\dot{x}+a(t, x) x=g(t, x) u(t) \quad \text { a.e. on } T, \\
x(0)=-x(b), \\
u(t) \in \operatorname{Ext} U(t, x(t)) \quad \text { a.e. on } T .
\end{array}\right.
$$

The hypotheses on the data (10) are the following.

$H(a): a: T \times R^{N} \rightarrow R^{+}, g: T \times R^{N} \rightarrow R$ are Carathéodory functions such that for almost all $t \in T$

$$
\begin{aligned}
& 0<\theta_{1} \leq a(t, x) \leq \theta_{2}, \\
& |g(t, x)| \leq \eta_{1}(t)+\eta_{2}(t)|x|^{\alpha},
\end{aligned}
$$

with $\theta_{1}, \theta_{2}>0,0<\alpha<1, \eta_{1}(t) \in L_{+}^{2}(T), \eta_{2}(t) \in L^{\infty}(T)$.

$H(U): U: T \times R^{N} \rightarrow P_{k c}\left(R^{N}\right)$ is a multifunction such that

(i) for all $x \in R^{N}, t \rightarrow U(t, x)$ is measurable;

(ii) for all $t \in T, x \rightarrow U(t, x)$ is $h$-continuous;

(iii) for almost all $t \in T$ and all $x \in R^{N},|U(t, x)| \leq \gamma$, with $\gamma>0$.

Let $A: T \times R^{N} \rightarrow R^{N}$ be the operator defined by $A(t, x)=a(t, x) x$. Evidently, using hypothesis $H(a)$, it is straightforward to check that $A$ satisfies hypothesis $H(A)$. Also, let $F: T \times R^{N} \rightarrow P_{k c}\left(R^{N}\right)$ be defined by

$$
F(t, x)=\left\{y \in R^{N}: y(t)=g(t, x(t)) u(t), u(t) \in U(t, x(t)) \text { a.e. on } T\right\} .
$$

Using hypotheses $H(a)$ and $H(U)$, it is straightforward to check that $F$ satisfies hypothesis $H(F)_{1}$.

Rewrite problem (10) in the following equivalent evolution inclusion form:

$$
\left\{\begin{array}{l}
\dot{x}+A(t, x(t)) \in \operatorname{Ext} F(t, x) \quad \text { a.e. on } T, \\
x(0)=-x(b) .
\end{array}\right.
$$

We can apply Theorem 3.1 on problem (11) and obtain the following.

Theorem 4.1 If the hypotheses $H(a)$ and $H(U)$ hold, then problem (10) has a solution $x \in W^{1,2}\left(I ; R^{N}\right)$. 


\section{Competing interests}

The authors declare that they have no competing interests.

\section{Authors' contributions}

All authors contributed equally and significantly in writing this paper. All authors read and approved the final version.

\section{Author details}

${ }^{1}$ School of Aerospace Engineering, Beijing Institute of Technology, Beijing, 100081, P.R. China. ${ }^{2}$ Department of Mathematics, Bohai University, Jinzhou, 121013, P.R. China. ${ }^{3}$ Center of Teaching Innovation and Evaluation, Bohai University, Jinzhou, 121013, P.R. China. ${ }^{4}$ Department of Foundation, Aviation University of Air Force, Changchun, 130022, P.R. China.

\section{Acknowledgements}

The first author was also supported by NSFC Grant 11172036. The authors are also thankful to the referee for careful reading of the paper and valuable comments.

Received: 5 September 2013 Accepted: 24 February 2014 Published: 12 Mar 2014

\section{References}

1. Okochi, $\mathrm{H}$ : On the existence of periodic solutions to nonlinear abstract parabolic equations. J. Math. Soc. Jpn. 40, 541-553 (1988)

2. Aizicovici, S, McKibben, M, Reich, S: Anti-periodic solutions to nonmonotone evolution equations with discontinuous nonlinearities. Nonlinear Anal. 43, 233-251 (2001)

3. Aizicovici, S, Reich, S: Anti-periodic solutions to a class of non-monotone evolution equations. Discrete Contin. Dyn Syst. 5, 35-42 (1999)

4. Aftabizadeh, AR, Aizicovici, S, Pavel, NH: Anti-periodic boundary value problems for higher order differential equations in Hilbert spaces. Nonlinear Anal. 18, 253-267 (1992)

5. Chen, YQ: Anti-periodic solutions for semilinear evolution equations. J. Math. Anal. Appl. 315, 337-348 (2006)

6. Franco, D, Nieto, JJ, O’Regan, D: Anti-periodic boundary value problem for nonlinear first order ordinary differential equations. Math. Inequal. Appl. 6, 477-485 (2003)

7. Okochi, $\mathrm{H}$ : On the existence of anti-periodic solutions to a nonlinear evolution equation associated with odd subdifferential operators. J. Funct. Anal. 91, 246-258 (1990)

8. Chen, YQ, Cho, YJ, O'Regan, D: Anti-periodic solutions for evolution equations. Math. Nachr. 278, 356-362 (2005)

9. Liu, Q: Existence of anti-periodic mild solutions for semilinear evolution equations. J. Math. Anal. Appl. 377, 110-120 (2011)

10. Liu, ZH: Anti-periodic solutions to nonlinear evolution equations. J. Funct. Anal. 258, 2026-2033 (2010)

11. Wang, Y: Antiperiodic solutions for dissipative evolution equations. Math. Comput. Model. 51, 715-721 (2010)

12. Li, GC, Xue, XP: On the existence of periodic solutions for differential inclusions. J. Math. Anal. Appl. 276, 168-183 (2002)

13. Xue, $X, Y$, J: Periodic solutions for semi-linear evolution inclusions. J. Math. Anal. Appl. 331, 1246-1262 (2007)

14. Xue, XP, Cheng, Y: Existence of periodic solutions of nonlinear evolution inclusions in Banach spaces. Nonlinear Anal., Real World Appl. 11, 459-471 (2010)

15. Zhang, JR, Cheng, Y, Yuan, CQ, Cong, FZ: Properties of the solutions set for a class of nonlinear evolution inclusions with nonlocal conditions. Bound. Value Probl. 2013, 15 (2013)

16. Zeidler, E: Nonlinear Functional Analysis and Its Applications, vol. II. Springer, Berlin (1990)

17. Bressan, A, Colombo, G: Extensions and selection of maps with decomposable values. Stud. Math. 90, 69-86 (1988)

18. Tolstonogov, A: Continuous selectors of multivalued maps with closed, nonconvex, decomposable values. Russ. Acad. Sci. Sb. Math. 185, 121-142 (1996)

19. Cheng, Y, Cong, FZ, Hua, HT: Anti-periodic solutions for nonlinear evolution equations. Adv. Differ. Equ. 2012,165 (2012)

20. Donchev, T, Farkhi, E: Stability and Euler approximation of one-sided Lipschitz differential inclusion. SIAM J. Control Optim. 36(2), 780-796 (1998)

21. Donchev, T: Qualitative properties of a class differential inclusions. Glas. Mat. 31(51), 269-276 (1996)

22. Hu, S, Papageorgiou, NS: On the existence of periodic solution for nonconvex valued differential inclusions in $R^{N}$. Proc. Am. Math. Soc. 123, 3043-3050 (1995)

10.1186/1029-242X-2014-111

Cite this article as: Zhang et al.: Anti-periodic extremal problems for a class of nonlinear evolution inclusions in $R^{N}$. Journal of Inequalities and Applications 2014, 2014:111 